\title{
BokSmart: Pre-participation screening of rugby players by coaches based on internationally accepted medical standards
}

\author{
J S Patricios (MB BCh, MMedSci (Sheffield), FACSM, FFSEM (UK)) ${ }^{1}$ \\ R M Collins (MB BCh, MSc (Sports Med)) \\ ${ }^{1}$ Sports Physician, Johannesburg, Extraordinary Lecturer, Department of Sports Medicine, University of Pretoria, and Consultant, South Afri- \\ can Rugby's BokSmart Injury Prevention Programme \\ ${ }^{2}$ Sports Physician in private practice, Johannesburg, Team Physician, Lions Rugby Union, and Lecturer, Department of Sports Medicine, \\ University of Pretoria
}

\begin{abstract}
s
A comprehensive medical history forms a significant part of any medical assessment or screening. In the athlete, pre-participation screening is aimed at determining those aspects of personal and family history that place the participant at greater risk of sudden death, serious illness or musculoskeletal injury. In rugby union, where the incidence of head and neck injuries is higher than in other sports, emphasis needs to be placed on screening for potential risk factors for neurological injury. In a South African rugby environment, pre-season medical screening is not standard and indeed rarely practised. In most club and school settings, the rugby coach may well be the person most in contact with players and therefore in the best position to conduct an initial screening. This article reviews the relevant literature pertinent to such a guideline.
\end{abstract}

\section{Introduction}

In any medical consultation, the history serves as a very important initial assessment of the patient's presenting complaint or condition. Pre-participation evaluations (PPEs) are largely designed for medically qualified personnel to screen for players who may be at risk of illness or injury. ${ }^{1-12}$ The most recent example of such a template is FIFA's Pre Competition Medical Assessment (PCMA) ${ }^{13}$ used to screen all participants before the recent FIFA 2010 World Cup. Much of the emphasis is on cardiovascular disease, as this is the largest

\section{CORRESPONDENCE:}

\section{Dr Jon Patricios}

Morningside Sports Medicine

PO Box 1267

Parklands 2121

Tel: +27 118839000

Fax: +27 114428233

E-mail: jpat@mweb.co.za cause of sudden death in young athletes. ${ }^{14}$ In South African rugby, particularly at school and community club level, pre-participation screening is rarely conducted owing to a lack of resources and skills. In designing a pre-participation screen for coaches, the challenge is to be able to 'red flag' potentially serious cardiovascular risk factors as well as musculoskeletal and neurological risks pertinent to a collision sport such as rugby, using easily understood questions that cast the screening net wide enough to determine who should be formally medically assessed (see Table I). ${ }^{15}$

\section{Cardiovascular screening}

Most athletes are healthy. Only $3-13 \%$ require further evaluation, and the disqualification rate for 10 million annual examinations is less than $1 \%$. ${ }^{10}$ The overall rate of sudden death in male athletes younger than 35 years is quite low, approximately 0.75 per 100000 participants per year. ${ }^{14}$ Congenital cardiac anomalies account for most sudden deaths in these patients. The most common anomalies are hypertrophic cardiomyopathy and coronary artery anomalies. ${ }^{14,16}$ The most common coronary abnormality is a left main coronary artery originating off the right sinus. Myocarditis, rupture of the aorta, arrhythmogenic right ventricular dysplasias, idiopathic left ventricular hypertrophy, aortic stenosis and premature coronary artery disease account for most of the remaining fatalities. ${ }^{2}$

Cardiovascular screening is regarded as the most important part of a PPE because of the potential for sudden death in athletes with undiagnosed heart disease. ${ }^{17,18}$ Personal and family histories of cardiovascular illness have been shown to be more sensitive screens than a physical examination, revealing $64-78 \%$ of conditions that could prohibit or alter sports participation. ${ }^{5,12}$ Nevertheless, screening on the basis of symptoms is certainly not comprehensive and hypertrophic cardiomyopathy, the most common cause of death among 12 - 32-year-old athletes on the field, may not produce symptoms before sudden death. ${ }^{19}$ Hypertrophic cardiomyopathy is a heterogeneous group of disorders acquired through autosomaldominant transmission with incomplete penetrance, and signs and symptoms may not become manifest until early adulthood. ${ }^{20}$ Detecting persons with Marfan syndrome before they participate in sports is important because the defective aortic media can rupture during basketball, volleyball and, presumably, other sports activities. ${ }^{21}$ Where a layperson such as a coach is performing the screen and has limited experience in screening for potential risks such as Marfan syndrome and does not have the benefit of a physical examination 
TABLE I. Cardiovascular screening history for preparticipation examinations

\section{Critical questions ${ }^{14}$}

Exertional chest pain or discomfort, or shortness of breath?

Exertional syncope or near-syncope, or unexpected fatigue?

Past detection of cardiac murmur or systemic hypertension?

Known family history of hypertrophic cardiomyopathy, other cardiomyopathies, long QT syndrome, Marfan syndrome, significant dysrhythmias?

Family history of premature death or known disabling cardiovascular disease in a first- or second-order relative younger than 50 years? (More concern if younger than 40 years)

or ancillary studies (e.g. ECGs, echocardiogram) to supplement the history, the risks may be greater.

\section{Neurological screening}

Head and neck injuries account for the largest proportion of catastrophic injuries in South African rugby ${ }^{22}$ and should therefore be adequately screened for in any PPE.

\section{Neck injuries}

A report of burning pain, weakness, numbness or tingling in all four or only the upper extremities raises concern of cervical spine impingement. Possible aetiologies for this condition would include atlantoaxial instability, congenital fusions and disk herniations. ${ }^{14}$

\section{Nerve injuries}

'Burners' or 'stingers' are usually secondary to a brachial plexus stretch or cervical root irritation. The athlete should be free of any neck or radicular pain, and have full range of motion and strength in all movements of the cervical spine before returning to sports participation. ${ }^{23}$ Recurrent episodes require referral for cervical radiographic and/or neurophysiological studies before clearance.

\section{Concussion}

Pre-participation examinations for neurological problems such as concussion are extremely difficult, as most concussions recover fully and leave no residual indicators. In addition, concussions sustained in contact and collision sports may simply reflect an athlete's level of exposure to the sport rather than an underlying intrinsic risk factor. ${ }^{3}$

The definition of concussion has been broadened to include any trauma-induced alteration in mental state (Table II) and does not necessarily include loss of consciousness or amnesia as in previous definitions. $^{23-26}$ The range of symptoms possibly associated with concussion should be made clear to athletes, many of whom may not have recognised or appreciated their significance.

Risks of playing while the athlete has prolonged concussion include exacerbation or prolongation of symptoms of the postconcussion syndrome. This is of particular significance in young players exposed to a learning environment. The second-impact syndrome is a less common but far more catastrophic consequence of unrecognised or poorly managed concussion. A second blow (even a relatively mild impact) to a brain that has not yet recovered from a previous blow may result in loss of autoregulation. ${ }^{27,28}$ Any player who is still symptomatic from a concussive blow should not be exercising and definitely not participating in contact or collision sports. ${ }^{24,26,29}$ Finally, there is evidence of the cumulative effect of concussions, particularly where these injuries may not have been recognised or managed appropriately. ${ }^{30}$ Coaches detecting any symptoms, recent history of concussion or multiple concussions in a player should ensure that the player seeks appropriate medical advice.

To help to mitigate the difficulties in detecting concussion risk in collision sport, it is recommended that an additional and more extensive baseline screening of symptoms, previous episodes and comorbid neurological and psychological risk factors be conducted. ${ }^{8}$ In addition, the emergence of computerised neuropsychological testing, where accessible, provides the player with an assessment that may give insight into cognitive compromise related to previous injuries and serve as a baseline measure against which the consequences of further concussions may be measured. ${ }^{24,26,29}$

\section{Convulsive disorders}

Guidelines from the American Academy of Pediatrics ${ }^{31}$ clear young athletes with well-controlled convulsive disorders for participation in conventional school-sponsored sports. However, in a sport entailing higher risk, including rugby union, neurological consultation should be considered. Athletes with poorly controlled seizures should be withheld from contact or collision sports. ${ }^{14}$

\section{Musculoskeletal injuries}

Most studies have shown that musculoskeletal findings are the major category of abnormalities leading to restriction from sports activities. $^{11}$ The most common musculoskeletal injury to restrict an athlete from activity is a knee injury, followed by an ankle injury. ${ }^{4}$ In musculoskeletal injuries, the chance of re-injury is high without proper rehabilitation. ${ }^{16}$ Specific examples include patella and shoulder dislocations. ${ }^{11}$ However, this category of injury is unlikely to be catastrophic and, therefore, in the interests of efficiency, should receive no more than a mention in a coach's PPE.

\section{Other medical conditions}

Leading causes of non-traumatic, non-cardiac sports death are exertional hyperthermia, followed by exertional rhabdomyolysis and status asthmaticus. 32

\section{Current infections}

Conditions such as influenza or gastroenteritis affecting the player at the time of questioning should preclude him or her from training and appropriate medical care should be sought to avoid the risk of myocarditis and pericarditis. $9,10,14$

\section{Exercise-induced asthma}

Status asthmaticus is one of the non-traumatic causes of death in high-school and college athletes. However, the incidence in survey populations is only four deaths in 30 million athletes. ${ }^{32}$ Evidence of exercise-induced asthma is sought in the pre-participation examination so that medical prophylaxis (typically with a beta agonist) can be implemented, not to disqualify the athlete.

\section{Heat-related illness}

Physicians can screen for a tendency toward exertional hyperthermia by asking about a previous history of heat-related illness. Ath- 


\begin{tabular}{|llll|}
\hline \multicolumn{2}{|c|}{ TABLE II. Symptoms and } & signs of concussion ${ }^{28}$ \\
\hline Physical & Cognitive & Emotional & Sleep \\
Headache & Poor & Depression & Drowsiness \\
Photophobia & concentration & Irritability & Insomnia \\
Dizziness & Problems & Mood swings & Sleeping more \\
Phonophobia & remembering & Aggressive- & $\begin{array}{l}\text { Difficulty get- } \\
\text { ting to sleep }\end{array}$ \\
& & ness & \\
Nausea & Feeling & & \\
Numbness/ & 'foggy' & & \\
tingling & Feeling & & \\
Vomiting & 'slowed down' & & \\
Fatigue & & & \\
Visual & & & \\
changes & & \\
Balance & & \\
Problems & & \\
\hline
\end{tabular}

letes with this condition are usually allowed to participate in sports, but temperature extremes must be avoided and appropriate means of cooling such as breaks in play and iced towel-downs should be followed.

\section{Sickle cell trait}

The American Academy of Pediatrics and the National Collegiate Athletic Association recommend that persons with sickle cell trait be allowed to participate in sports without any restrictions. ${ }^{31}$ There is evidence that persons with sickle cell trait have increased susceptibility to exertional rhabdomyolysis, with the potential for renal failure and death. Patients with sickle cell trait should be counselled about appropriate hydration and acclimatisation to reduce risks.

Rugby players should note that high exertion and contact or collision sports are generally contraindicated in patients with sickle cell disease, even if appropriate hydration can be ensured.

\section{Solitary organs}

Whether athletes with one paired organ, especially one kidney, should participate in sport, particularly collision sports, is a topic of controversy. All such patients need to understand the risks so they can make an informed decision. No contact or collision sports are allowed if a single kidney is polycystic or abnormally located. ${ }^{1}$

When an athlete has only one functional eye (with less than 20/40 corrected visual acuity), further evaluation by an ophthalmologist is recommended. ${ }^{33}$ These athletes can participate only in sports that permit the use of protective eyewear (such as swimming, track and field, and gymnastics) and do not involve projected objects. Wrestling, boxing and martial arts are contraindicated sports and, by inference, rugby must also be regarded as risk sport for these individuals.

The only modification for an athlete with one testicle is the use of a protective cup or 'box' during contact sports. The chance of injury and the subsequent possibility of loss of fertility should be mentioned in counselling. ${ }^{1}$

\section{Other benefits of screening}

Enquiries about medication use may have several benefits. Firstly, medications may have a direct influence on performance, e.g. anti- histamines may cause drowsiness. Secondly, medications may require Therapeutic Use Exemption clearance or be banned in competition. This should be brought to the player's attention. Thirdly, it may serve as a means of determining a medical condition that the athlete did not feel was worth mentioning because it is such an inherent part of his/her life, e.g. asthma, diabetes or hypertension. Finally, the athlete may mention additional supplements (legal and illegal) that are being consumed. ${ }^{14,34}$

A further benefit of PPE is the opportunity it affords the coach to gain some insight into a player on a one-to-one basis. Although not specifically targeted in most PPE questionnaires, issues not directly related to sport but affecting the athlete's lifestyle such as smoking, alcohol and drug use may emerge during the questioning, affording counselling (formal or informal) to be given in these areas.

\section{Ethical considerations}

The coach, as a lay person, should not necessarily be privy to medical information that the player may regard as confidential. Hence, an option should be included in the survey that allows the player to share this information with a medical practitioner in private, particularly if this information, e.g. HIV infection, has implications for the player and others' participation in exercise.

\section{Fitness assessment}

Although the coach does not have the advantage of a physical examination to complement the history, he/she is afforded the benefit of seeing the athlete under conditions of physical stress when training. This can be utilised as a screening tool in itself and a sort of field 'stress test'. In particular, players who fail to cope with exercise that their peers find reasonable, those who show a marked decrease from previous levels of performance, those who describe symptoms during or following exercise or those who appear hindered by injury should be referred for medical evaluation. $9,11,14$

\section{Conclusion}

In an amateur sporting environment where pre-participation screening is sparingly utilised, the use of a screening tool administered by rugby coaches could significantly and positively impact on the detection and reduction of potentially catastrophic illness and injury. A review of the relevant literature shows the player's medical and family history to be the most important part of the screen and is therefore within the parameters of such a questionnaire. In particular this would aim at detecting a higher risk for cardiac-related sudden death, concussion and other neurological injuries.

\section{Acknowledgements}

This paper was commissioned by the BokSmart programme - a national programme sponsored by ABSA and implemented on behalf of the South African Rugby Union and the Chris Burger/Petro Jackson Players' Fund. The goal of the programme is to teach safe and effective techniques, which will reduce the incidence and severity of injury, make the game safer for all involved and improve rugby performance.

\section{References}

1. Abdulla A. The pre-participation of athletes. Middle Eastern Journal of Family Medicine 2007;5(4):17-20.

2. American Heart Association. Cardiovascular preparticipation screening of competitive athletes. Med Sci Sports Exerc 1996;28:1445-1452. 
3. Fuller C, Ojelade E. Preparticipation medical evaluation in professional sport in the UK: theory or practice? Br J Sports Med 2007;41:890-896.

4. Grafe MW, Paul GR, Foster TE. The preparticipation sports examination for high school and college athletes. Clin Sports Med 1997;16:569-591.

5. Krowchuk DP. The preparticipation athletic examination: a closer look. Pediatr Ann 1997;26:37-49.

6. Maron BJ, Isner JM, McKenna WJ. 26th Bethesda Conference: recommendations for determining eligibility for competition in athletes with cardiovascular abnormalities. Med Sci Sports Exerc 1994;26:S261-267.

7. Maron BJ, Thompson PD, Puffer JC, et al. Cardiovascular preparticipation screening of competitive athletes. Circulation 1996;94:850-856.

8. McCrory P. Preparticipation assessment for head injury. Clin J Sport Med 2004;14:139-144.

9. Morphet JAM. Screening for sudden cardiac death in adolescent athletes. Perspect Cardiol 2001;17(8):37-47.

10. Smith DM. Preparticipation Physical Evaluation. 2nd ed. Minneapolis: Physician and Sports Medicine, 1997:1-46.

11. Smith J, Laskowski ER. The preparticipation physical examination. Mayo Clin Proc 1998;73:419-429.

12. Wappes JR (executive editor). Preparticipation Physical Evaluation. 3rd ed. Minneapolis: McGraw Hill, 2005.

13. FIFA PCMA Form. Available at: http://www.fifa.com $/ \mathrm{mm} /$ document/ afdeveloping/medical/01/07/26/86/fifapcmaform.pdf (accessed: 17 July 2010).

14. Kurowski K, Chandran S. The preparticipation athletic evaluation. Am Fam Physician 2000;61:2683-2690, 2696-2698

15. Holtzhausen L, Schwellnus M, Jakoet I, Pretorius A. Pre-season assessment of South African players in the 1999 rugby Super 12 competition. S Afr J Sports Med 2002;9:15-21.

16. Abbott HG, Kress JB. Preconditioning in the prevention of knee injuries Arch Phys Med Rehabil 1969;50:326-333.

17. Basilico FC. Cardiovascular disease in athletes. Am J Sports Med 1999;27:108-121.

18. Maron BJ. Hypertrophic cardiomyopathy. Lancet 1997;350:127-133.

19. Maron BJ, Casey SA, Poliac LC, Gohman TE, Almquist AK, Aeppli DM Clinical course of hypertrophic cardiomyopathy in a regional United States cohort. JAMA 1999;281:650-655.

20. Lerakis S, Sheahan RG, Stouffer GA. Hypertrophic cardiomyopathy. Am J Med Sci 1997:314:324-329.
21. Gott VL, Pyeritz RE, Magovern GJ, Cameron DE, McKusick VA. Surgical treatment of aneurysms of the ascending aorta in the Marfan syndrome. N Engl J Med 1986;314:1070-1074.

22. Noakes T, Du Plessis M. Common rugby injuries, including anatomical sites and mechanisms of injury. In: Rugby Without Risk. Pretoria: JL van Schaik, 1996:45-86.

23. Cantu RC, Bailes JE, Wilberger JE. Guidelines for return to contact or collision sport after a cervical spine injury. Clin Sports Med 1998;17:137146

24. Aubry M, Cantu R, Dvorak J, et al. Summary and agreement statement of the First International Conference on Concussion in Sport, Vienna 2001. Recommendations for the improvement of safety and health of athletes who may suffer concussive injuries. Br J Sports Med 2002;36:6-10.

25. Cantu RC. Cerebral concussion in sport. Management and prevention. Sports Med 1992;14:64-74.

26. Herring SA, Bergfield JA, Boland A, et al. ACSM Team Physician Consensus Statement: Concussion (Mild Tarumatic Brain Injury) and the Team Physician. Med Sci Sport Exerc 2006;2:395-399.

27. Cantu RC. Second-impact syndrome. Clin Sports Med 1998;17:37-44.

28. Gioia G, Collins M. Acute Concussion Evaluation (ACE). Heads Up: Brain Injury In Sport. Your Practice Tool Kit. Center for Disease Control. www. cdc.gov/ncipc/pub-res/tbi_toolkit/tbi/ACE (modified June 2007).

29. Guskiewicz KM, Bruce SL, Cantu RC, et al. National athletic trainers position statement on the management of sports-related concussion. Journal of Athletic Training 2004;39:278-295.

30. Collins MW, Lovell MR, Iverson GL, Cantu R, Maroon J, Field M. Cumulative effects of concussion in high school athletes. Neurosurgery 2002;51:1175-1181.

31. American Academy of Pediatrics Committee on Sports Medicine Recommendations for participation in competitive sports. Pediatrics 1988;81:737-739

32. Van Camp SP, Bloor CM, Mueller FO, Cantu RC, Olson HG. Non-traumatic sports death in high school and college athletes. Med Sci Sports Exerc 1995;27:641-647.

33. Stock JG, Cornell FM. Prevention of sports-related eye injury. Am Fam Physician 1991;44:515-520.

34. Welder AA, Melchert RB. Cardiotoxic effects of cocaine and anabolic-androgenic steroids in the athlete. J Pharmacol Toxicol Methods 1993;29:61-68 